\title{
An Exploration of Sexual Desire and Sexual Activities of Women with Psychosis
}

\author{
Philippe Huguelet $\cdot$ Sylvia Mohr $\cdot$ Céline Miserez $\cdot$ \\ Patrizia Castellano Christian Lutz • Maria Boucherie • \\ Michal Yaron • Nader Perroud · Francesco Bianchi Demicheli
}

Received: 26 November 2013/Accepted: 15 July 2014/Published online: 27 July 2014

(C) Springer Science+Business Media New York 2014

\begin{abstract}
Sexual disturbances in patients with severe mental disorders can be related to medication, to psychological issues such as self-stigma and anhedonia, but also to the social context. This research aims to gain knowledge of desire and sexual practices in women suffering from schizophrenia. Women outpatients suffering from schizophrenia were compared with healthy women. Contrary to previous research, women with schizophrenia featured dyadic and individual desire similar to women of comparable age. Yet, only half of women with psychosis had sexual practice, either alone or with a partner. They were less satisfied with their activity, both in terms of function and psychological issues such as sexual self-esteem. Keeping into account the fact that most clinicians avoid this topic, this finding underscores the stigmatization these women suffer from, which prevents the opportunity of a possible improvement in this important interpersonal domain.
\end{abstract}

Keywords Psychosis - Schizophrenia - Women - Sexual desire $\cdot$ Sexual function

P. Huguelet $(\varangle) \cdot$ S. Mohr $\cdot$ C. Miserez $\cdot$ P. Castellano .

C. Lutz $\cdot$ M. Boucherie $\cdot$ N. Perroud

Service of General Psychiatry, Department of Mental Health and Psychiatry, University Hospitals of Geneva, Secteur Eaux-Vives,

Rue du 31-Décembre 8, 1207 Geneva, Switzerland

e-mail: Philippe.huguelet@hcuge.ch

M. Yaron · F. Bianchi Demicheli

Psychosomatic Gynecology, Department of Gynecology,

University Hospitals of Geneva, Geneva, Switzerland

\section{Introduction}

Among the issues that are pertinent for the care of individuals suffering from mental disorders such as schizophrenia, the intimate sexual and interpersonal needs remain marginalized and neglected (McCann 2010; Ostman and Bjorkman 2013). Thornicroft et al. (2009) showed that patients with schizophrenia experienced negative discrimination in $27 \%$ of cases for intimate or sexual relationships. Also, two-third of them feature self-stigmatization in close relationships (Brohan et al. 2013). This issue should be a justifiable concern for mental health professionals, particularly when considering that $75 \%$ of patients may be willing to address it with their clinicians (Lewis and Scott 1997). In this perspective, Seeman (2013) underscored that all women of childbearing age should be engaged in discussions about contraception and should be treated as potential new mothers.

Desire has been defined as "...the sum of the forces that lean us toward and away from sexual behavior..." (Levine 2003). Sexual desire combines drive (biological), motivation (i.e. individual and relationship psychology), and wish (i.e. "the cultural component that reflects values, meanings and rules about sexual expression") components. Sexual desire is characterized by an interest or an increase in the frequency and the intensity of sexual thoughts/fantasies (either spontaneous or in response to erotic stimuli) and corresponds more to an urge that impels individuals to interact with others and initiate and/or to respond to sexual stimulation (Basson 2006, 2005; Giles 1958; Kaplan 1995).

Few papers addressed sexuality and sexual desire in patients with severe mental disorders, most of them with methods involving global statistics rather than protocols investigating subjective experience (Kelly and Conley 2004). Sexual disturbances can be related to medication 
(e.g. antipsychotic medications) (Seagraves 1992), but also to concomitant disorders such as diabetes. Yet sexual dysfunction can also be related to "psychosocial" issues such as lower self-esteem, fewer relationships and negative symptoms such as anhedonia (McCann 2010). MacDonald et al. (2003) showed with a self-completed survey that sexual dysfunction occurred in $82 \%$ of men and $96 \%$ of women with schizophrenia, i.e. a higher score as compared with subjects drawn out of a general population. Patients may experience difficulties in the areas of sexual desire, sexual arousal and orgasm (Fan et al. 2007). Intimate relationships may also be affected (McCann 2010; Neumann and Frasch 2001; Raja and Azzoni 1993). Peitl et al. (2009) explored sexual self-perception among acute and chronic patients with schizophrenia, compared with healthy controls, and showed that patients exhibited higher negative emotionality, likely to emerge from one's experience of sexual incompetence, abandonment and isolation. These difficulties and dissatisfactions interfere with quality of life (Bushong et al. 2011), but can as well lead to various complications such as risky behavior, unplanned pregnancies (Dickson and Dawson 1998), contamination by HIV (Johnson 1994) and abuse, both as victim or perpetrator (Friedman and Harrison 1984; Miller and Finnerty 1996). Recently Ostman and Bjorkman (2013) performed qualitative analyses of the interviews of five women with schizophrenia who were in an intimate relationship at that time. They pointed the "bad or non-existent sexual relationships" experienced by these women, who were often uncertain about their capacities for sexual activities and furthermore failed to achieve satisfaction during intercourse.

Gender plays obviously a major role in sexual function and intimate relationship, both in biological (e.g. hormones) and socio-psychological perspectives. Gender effect has thus to be taken into account in studies investigating sexuality and desire. That led us to limit our scope on women with psychosis. As described above, beyond statistics on prevalence of sexual dysfunction, there is only limited research addressing specifically sexuality in women with psychosis. In particular, research on sexual desire and sexual fantasies in women with psychosis is scarce. This lack of literature is likely to foster stigmatization of women with psychosis. Indeed clinicians may adopt a stance considering that this possible lack of sexual drive is not worth an intervention, due to the widespread deficit reported.

In this context the goal of this research was to gain knowledge of sexual practices and desire of women with psychosis, in order to get more clinical clues as to why and how to intervene. A comparison of a group of women suffering from schizophrenia and a group of women of comparable age consulting in a gynecologic facility was performed. An in-depth investigation involving specific questionnaires on sexual desire, sexual function and past sexual, physical and psychological abuse was performed. The hypothesis was that women with psychosis would feature altered sexual desire and sexual function, that being correlated with past trauma and symptoms' severity.

\section{Methods}

\section{Subjects}

This study took part in two public facilities: an outpatient psychiatric clinic where patients with psychosis are treated, and an outpatient gynecologic clinic where women go to perform their annual gynecological check-up. Participants from the "psychiatric" group were stabilized ambulatory patients aged from 18 to 50 who met the ICD-10 (World Health Organization 1993) diagnostic criteria for schizophrenia or other chronic psychoses.

To avoid selection bias related to sexual and relational characteristics of subjects, we first provided psychiatrists of the outpatient clinics with information about the research. After that, each clinician's list of patients was screened by the research investigators in order to identify patients eligible for the research. Then psychiatrists asked every consecutive eligible patient to enroll. Refusals were $50 \%$ of the eligible patients, that being related to uneasiness or unwillingness to speak about sexuality, or lack of interest. Healthy controls were randomly recruited in the waiting room of the public gynecologic facility. Refusal rate was of $66 \%$ for this group.

The study was approved by the ethics committee of the University Hospitals of Geneva. Subjects received detailed information about the study and gave their written consent.

\section{Procedure}

The Mini-International Neuropsychiatric Interview (Sheehan et al. 1998) was administered to screen for current or past history of formally diagnosable psychiatric disorders and substance misuse in both groups, in order to confirm a psychotic disorder in the psychiatric group and to exclude any psychiatric condition in the control group. Patients were administered the Positive and negative syndrome scale (PANSS) (Kay et al. 1992), as well as the Medication Adherence Rating Scale (MARS), (Thompson et al. 2000), the Morningside Rehabilitation Status Scale (MRSS) (Affleck and McGuire 1984) and the GAF (Goldman et al. 1992) for measuring social adaptation. The Childhood Trauma Questionnaire (CTQ), a self-report questionnaire that examines five types of trauma (sexual abuse, physical abuse, physical neglect, emotional abuse and emotional 
neglect), was used to assess childhood traumatic experience (Bernstein et al. 2003).

\section{Sexual Desire and Function}

Sexual desire was measured with the Sexual Desire Scale (SDS) (Tremblay and Roussy 2002). The SDS is a 20-item, self-report measure of two dimensions of sexual desire, i.e. the dyadic sexual desire (sexual desire to have a sexual interaction with someone, such as "I make sexual advances when I can see that my partner or a person that attracts me shows attention and interest in me.") and the individual sexual desire (sexual desire to have a solitary sexual activity); each dimension encompasses the subjective experience of feeling sexual desire, the search for sexual stimuli, and sexual fantasies and daydreams. Each item is rated on a scale from 1 to 5 ( $1=$ never, $2=$ rarely, 3 = occasionally, $4=$ often, $5=$ very frequently). Two items are reversed. The items are summed, so that higher scores correspond to higher sexual desire.

The Female Sexual Function Index (FSFI) is a 19-item, self-report measure of six different aspects of sexual function, i.e. sexual desire (measured by its frequency and its intensity), arousal, lubrication, orgasm, satisfaction and pain with items such as "over the past 4 weeks, how often did you feel sexual desire or interest?" (Rosen et al. 2000). Each item is rated on a scale ranging from 0 to 5 or 1 to 5 , where a score of 0 indicates that the respondent reported having no sexual activity in the past month. Individual domain scores can be obtained by adding the scores of the individual items that comprise the domain and multiplying the sum by the domain factor (i.e., .6 for desire, .3 for arousal and lubrication, and .4 for the other three domains). A full scale score can be obtained by adding the scores for the six domains.

The Multidimensional Sexuality Questionnaire (MSQ, Sperry and Gilbert 2005) is a 60-item, self-report measure of 12 psychological tendencies associated with sexuality : (1) sexual-esteem, defined as positive regard for and confidence in the capacity to experience one's sexuality in a satisfying and enjoyable way; (2) sexual-preoccupation, defined as the tendency to think about sex to an excessive degree; (3) internal-sexual-control, defined as the belief that the sexual aspects of one's life are determined by one's own personal control; (4) sexual-consciousness, defined as the tendency to think and reflect about the nature of one's sexuality; (5) sexual-motivation, defined as the desire to be involved in a sexual relationship; (6) sexual-anxiety, defined as the tendency to feel tension, discomfort, and anxiety about the sexual aspects of one's life; (7) sexual-assertiveness, defined as the tendency to be assertive about the sexual aspects of one's life; (8) sexual-depression, defined as the experience of feelings of sadness, unhappiness, and depression regarding one's sex life; (9) external-sexual-control, defined as the belief that one's sexuality is determined by influences outside one's personal control; (10) sexual-monitoring, defined as the tendency to be aware of the public impression which one's sexuality makes on others; (11) fear-of-sex, defined as a fear of engaging in sexual relations with another individual; and (12) sexual-satisfaction, defined as the tendency to be highly satisfied with the sexual aspects of one's life". Each item is rated on a scale ranging from A to E. A score of A indicates "Not at all characteristic of me" whereas a score of E indicates "very characteristic of me". Several items are reversed. The items on each subscale are summed, so that higher scores correspond to greater amounts of each tendency.

All scales have been shown to have good internal consistency and good reliability.

Sexual history was investigated through open questions concerning the first sexual experiences, traumatic sexual experiences, current intimate relationships, abortion, as well as gynecological care. Finally subjects were asked open questions about relationships (i.e. friendship, family, children and intimate) and religion, in order to assess in a more thorough perspective their relational context.

\section{Statistics}

Data were analyzed with the Statistical Package for the Social Sciences (SPSS) version 15. Univariate statistics were used to compare the variable distributions between females with psychosis and healthy controls (Chi square, Wilcoxon Rank Test). Associations between clinical and social measures and sexual measures were described through Spearman rank correlations.

\section{Results}

\section{General Assessment}

Sociodemographic and clinical characteristics of the sample are described in Table 1. Patients with psychosis were more frequently without a current partner, without children and without professional training/employment. Overall, they featured characteristics of stabilized patients, with quite low scores on PANSS and residual psychosocial deficits. For the 18 patients, neuroleptics were prescribed (five received clozapine as first medication, four received olanzapine, three risperidone; one received either clopentixol, amisulpride, sertindole or aripiprazole). Twelve (66\%) had a good adherence to medication according to the MARS, three a partial adherence, two low adherence, and one refused her medication. Sexual desire was not correlated with adherence on medication. 
Table 1 Sociodemographic characteristics of subjects
${ }^{a}$ GAF scores range from 1 (permanent danger) to 100 (exceptionnal performing in numerous areas)

b Positive and Negative Symptoms Scale

c MARS score range from 0 (no adherence at all) to 10 (full adherence)

${ }^{d}$ Morningside Rehabilitation Status Scale range from 0 (independence, activity, social integration, no symptoms) to 7 (hospitalisation, inactivity, social isolation, and disorganisation of everyday life due to symptoms)

\begin{tabular}{|c|c|c|c|c|c|c|c|}
\hline & \multicolumn{3}{|c|}{$\begin{array}{l}\text { Women with psychosis } \\
(\mathrm{n}=18)\end{array}$} & \multicolumn{2}{|c|}{$\begin{array}{l}\text { Controls } \\
(\mathrm{n}=21)\end{array}$} & \multirow[t]{2}{*}{ Stat. } & \multirow[t]{2}{*}{$d f \quad p$} \\
\hline & Mean & \multicolumn{2}{|c|}{ SD } & Mean & SD & & \\
\hline Age (years) & 39 & \multicolumn{2}{|c|}{7} & 37 & 9 & - & NS \\
\hline Body mass index & 26 & \multicolumn{2}{|c|}{6} & 23 & 3 & - & NS \\
\hline Duration of illness (years) & 14 & \multicolumn{2}{|c|}{7} & & & & \\
\hline Global assessment of functioning ${ }^{\mathrm{a}}$ & 50 & \multicolumn{2}{|c|}{15} & & & & \\
\hline \multicolumn{8}{|l|}{ PANSS $^{\mathrm{b}}$} \\
\hline Positive symptoms & 16 & \multicolumn{2}{|c|}{6} & & & & \\
\hline Negative symptoms & 15 & \multicolumn{2}{|c|}{8} & & & & \\
\hline General symptoms & 33 & \multicolumn{2}{|c|}{9} & & & & \\
\hline Total score & 63 & \multicolumn{2}{|c|}{19} & & & & \\
\hline Medication Adherence Rating Score ${ }^{c}$ & 7 & & & & & & \\
\hline $\operatorname{MRSS}^{\mathrm{d}}$ & & & & & & & \\
\hline Dependency & 2.7 & & & & & & \\
\hline Inactivity & 3.1 & & & & & & \\
\hline Social isolation & 2.5 & & & & & & \\
\hline Effects of current symptoms & 3.6 & & & & & & \\
\hline & $\mathrm{N}$ & $\%$ & $\mathrm{~N}$ & $\%$ & Stat. & $d f$ & $p$ \\
\hline Diagnosis & & & & & & & \\
\hline Schizophrenia & 11 & 61 & & & & & \\
\hline Schizoaffective disorder & 7 & 39 & & & & & \\
\hline Relational status with a sexual partner & 3 & 17 & 17 & 81 & 16.03 & 1 & $<.0001$ \\
\hline Civil status & & & & & & & \\
\hline Single & 9 & 50 & 9 & 43 & - & - & NS \\
\hline Married & 1 & 6 & 7 & 33 & & & \\
\hline Separated, divorced or widowed & 8 & 44 & 5 & 24 & & & \\
\hline Has child or children & 4 & 22 & 15 & 71 & 9.39 & 1 & .002 \\
\hline Living & & & & & & & \\
\hline Alone & 13 & 72 & 3 & 14 & 15.26 & 2 & .0004 \\
\hline With partner, and child if any & 1 & 6 & 11 & 52 & & & \\
\hline With other persons & 4 & 22 & 7 & 33 & & & \\
\hline Ethnicity & & & & & & & \\
\hline Caucasian & 12 & 67 & 18 & 86 & - & - & NS \\
\hline African & 2 & 11 & 1 & 5 & & & \\
\hline Asian & 2 & 11 & 0 & 0 & & & \\
\hline Mixed & 2 & 11 & 2 & 10 & & & \\
\hline Study/professional training & & & & & & & \\
\hline Without & 5 & 28 & 2 & 10 & 7.81 & 2 & .02 \\
\hline Professional training & 11 & 61 & 8 & 38 & & & \\
\hline University or superior study & 2 & 11 & 11 & 52 & & & \\
\hline Employed & 8 & 44 & 18 & 86 & 7.43 & 1 & .01 \\
\hline Nicotine dependency & 8 & 44 & 7 & 33 & - & - & NS \\
\hline
\end{tabular}

According to sexual history, no differences were found across groups concerning the mean ages of the menarche (mean $=13 \pm 2$ ), the age of the first sexual experience (mean $=16 \pm 5$ ), the age of the first experience with coitus (mean $=18 \pm 4$ ) (taking into account the fact that two women with psychosis were virgin), and the rate of voluntary abortion (23\%). Concerning gynecological care, most patients with psychosis declared to be regularly followed up, despite having a sexual partner less frequently. 


\section{Sexual Desire and Sexual Function}

Table 2 shows data on sexual desire and various dimensions of sexuality. No differences across groups were found for sexual desire as measured by the SDS, whether alone or with a partner. Sexual desire led less often women with psychosis to engage in sexual behaviors than healthy controls. Indeed, half of women with psychosis had no sexual practices (alone or with a partner) vs. $5 \%$ of control $\left(\chi^{2}=6.83, d f 1, p .01\right)$. Moreover, women with psychosis experienced impairment in various domains of their sexual function, with significantly less sexual arousal, lubrication, orgasm and satisfaction during sexual practices. No women in the sample experienced sexual pain (dyspareunia).

The Multidimensional Sexuality Questionnaire (MSQ) shows that women with psychosis featured more sexual

Table 2 Sexual dimensions and traumas

\begin{tabular}{|c|c|c|c|c|c|c|}
\hline & \multicolumn{2}{|c|}{ Women with psychosis $(\mathrm{n}=18)$} & \multicolumn{2}{|c|}{ Controls $(\mathrm{n}=21)$} & \multirow[t]{2}{*}{ Stat. } & \multirow[t]{2}{*}{$p$} \\
\hline & Mean & SD & Mean & SD & & \\
\hline \multicolumn{7}{|l|}{ Sexual Desire Scale (SDS) ${ }^{\mathrm{b}}$} \\
\hline 1. Dyadic sexual desire & 37.7 & 13.1 & 44 & 10 & -1.67 & .094 \\
\hline 2. Individual sexual desire & 16.8 & 7.3 & 17.9 & 4.4 & -.65 & .518 \\
\hline \multicolumn{7}{|l|}{ Female Sexual Function Index $(\mathrm{FSFI})^{\mathrm{c}}$} \\
\hline Desire & 3.4 & 1.5 & 3.8 & .8 & -.84 & .42 \\
\hline For women with sexual practices & $n=9$ & $50 \%$ & $n=19$ & $95 \%$ & & \\
\hline Arousal & 3.2 & 1.6 & 4.5 & 1.4 & -1.93 & .054 \\
\hline Lubrication & 2.8 & 2 & 5.5 & .9 & -3.2 & .001 \\
\hline Orgasm & 3 & 2.3 & 4.9 & 1.5 & -2.03 & .042 \\
\hline Satisfaction & 2.4 & 1.5 & 4.3 & 1.1 & -2.62 & .01 \\
\hline Total score & 15.2 & 7.6 & 24.8 & 27.7 & -4.27 & .04 \\
\hline \multicolumn{7}{|c|}{ Multidimensional Sexuality Questionnaire (MSQ) ${ }^{\mathrm{d}}$} \\
\hline Sexual-esteem & 5.9 & 5 & 11.5 & 4.2 & -3.19 & .0014 \\
\hline Sexual-preoccupation & 6.2 & 6 & 3.8 & 3.6 & -.99 & .322 \\
\hline Internal-sexual-control & 11.9 & 3.3 & 12.2 & 3.8 & -.75 & .452 \\
\hline Sexual-consciousness & 13.2 & 3.2 & 14.3 & 2.8 & -.93 & .352 \\
\hline Sexual-motivation & 7.6 & 6 & 11.8 & 4.4 & -2.42 & .016 \\
\hline Sexual-anxiety & 6.5 & 4.5 & 2.8 & 2.7 & -2.64 & .008 \\
\hline Sexual-assertiveness & 8.7 & 3.3 & 8.8 & 2.4 & -.52 & .605 \\
\hline Sexual-depression & 6.1 & 5.6 & 1.2 & 2.1 & -3.58 & .0003 \\
\hline External-sexual-control & 5.1 & 3.6 & 1.6 & 2.5 & -3.48 & .0005 \\
\hline Sexual-monitoring & 5.2 & 4.5 & 1.2 & 1.5 & -3.15 & .0016 \\
\hline Sexual-fear & 10.2 & 2.7 & 8.3 & 1.4 & -2.47 & .013 \\
\hline Sexual-satisfaction & 7.5 & 4.8 & 12.2 & 4.5 & -2.98 & .0029 \\
\hline Total score & 104 & 21 & 68 & 19 & -2.98 & $<.0001$ \\
\hline \multicolumn{7}{|c|}{ Childhood Trauma Questionnaire $(\mathrm{CTQ})^{\mathrm{a}}$} \\
\hline 1. Emotional abuse & 11.4 & 6 & 7.4 & 5.5 & -.8 & .44 \\
\hline 2. Physical abuse & 8.8 & 4.9 & 5.6 & 4.4 & -1.03 & .335 \\
\hline 3. Sexual abuse & 7.7 & 5 & 6.7 & 4 & -.97 & .443 \\
\hline 4. Emotional neglect & 12.2 & 3.8 & 10.5 & 3.9 & -1.39 & .174 \\
\hline 5. Physical neglect & 8.6 & 2.8 & 5.5 & 3.8 & -2.27 & .028 \\
\hline Total score & 48.6 & 13.4 & 41.8 & 15.5 & -1.68 & .094 \\
\hline
\end{tabular}

a SDS: Dyadic sexual desire score range from 14 (no desire) to 70 (very frequent desire); SDS: Individual sexual desire score range from 8 (no desire) to 40 (very frequent desire)

${ }^{\mathrm{b}}$ FSFI scores range from 1 (absence of the function) to 6 (very high level of the function); Total score range from 0 to 36

${ }^{\mathrm{c}}$ IMS scores range from 0 (absence of the dimension) to 20 (very high level of the dimension); total score range from 0 to 240 ; a higher score describe a higher sexual dysfunction

${ }^{\mathrm{d}}$ CTQ scores range from 5 (no trauma) to 25 (severe trauma); Total score range from 25 to 125 
alterations, with a lesser sexual-esteem (impaired sexual self-esteem for $65 \%$ of women with psychosis vs. $29 \%$ of healthy control); lesser sexual-motivation, defined as the desire to be involved in a sexual relationship (impaired sexual motivation: 59 vs. $19 \%$ ); greater sexual-anxiety (pathological sexual-anxiety for 18 vs. $0 \%$ ); greater sexual-depression, defined as the experience of feelings of sadness, unhappiness, and depression regarding one's sex life (pathological sexual-depression for 12 vs. $0 \%$ ); greater external-sexual-control, defined as the belief that one's sexuality is determined by influences outside one's personal control (yet no women reach a pathological score); greater sexual-monitoring, defined as the tendency to be aware of the public impression which one's sexuality makes on others (pathological sexual-monitoring for $6 \mathrm{vs}$. $0 \%$ ); greater sexual-fear (pathological sexual-fear for 41 vs. $0 \%$ ); and lesser sexual-satisfaction (impaired sexual satisfaction: 53 vs. $0 \%$ ).

\section{Sexual Desire, Sexual Function and Past Trauma}

The CTQ shows that patients displayed more physical neglect than controls [66 vs. $29 \%$ according to Bernstein's cut off criteria (Bernstein et al. 2003)]. During childhood, patients did not suffer from sexual abuse more often than the controls ( 39 vs. $24 \% ; \chi^{2}=1.88, d f 1, p .17$ ); yet from adolescence they lived far more often traumatic sexual experiences than the controls ( 56 vs. $5 \% ; \chi^{2}=12.35, d f 1$, $p$.00), as well when considering their whole life (67 vs. $24 \% ; \chi^{2}=7.24$, df $\left.1, p .01\right)$. For women with psychosis, there were no associations between traumatic sexual experiences during adult life and sexual desire, sexual functioning (IFS), and sexual dysfunction (IMS).

The associations between sexual desire, sexual dysfunction, and childhood trauma differed among women with psychosis and control subjects. Indeed for healthy women, the dyadic sexual desire was inversely correlated with sexual dysfunction (IMS total score $\rho=-.65$, $p<.001$ ), but not with sexual functioning (IFS). The sexual measures were not correlated with childhood trauma experiences; except for childhood sexual abuse which was correlated with sexual dysfunction (IMS $\rho=.62, p<.01$ ), and inversely with sexual functioning (IFS $\rho=-.50$, $p$.03), but not with sexual desires. For women with psychosis, there were no correlations between the three measures of desire and sexual dysfunction (IMS), nor sexual functioning (IFS). Concerning childhood trauma experiences (CTQ scores), the desire of women with psychosis was correlated with emotional abuse $(\rho=.48$ FSFI, $p<.05 ; \rho=.58$ individual SDS, $p<.05)$; and with sexual abuse $(\rho=.53$ FSFI, $p<.05 ; \rho=.58$ individual SDS, $p<.05 ; \rho=.58$ dyad SDS, $p<.01)$. Childhood traumatic experiences were not correlated with sexual functioning
(ISF), or the presence of sexual activities. However, emotional abuse was correlated with sexual dysfunction $(\rho=.54$ IMS, $p<.05)$.

The dyadic sexual desire was not associated with symptoms (PANSS), and psychosocial adaptation (GAF, MRSS). Individual sexual desire was associated with positive symptoms $(\rho=.53$ individual SDS, $p<.03$; desire FSFI $\rho=.51, p<.04)$, general symptoms $(\rho=.50$ individual SDS, $p<.04$ ), and dependence (MRSS dependence scale: desire FSFI $\rho=.70, p<.01$ ).

For all women, there was no association between sexual desire, age, and BMI.

\section{Other Data on Social Involvement}

Table 3 shows data on social relationships and religion/ spirituality, in order to give a more general view on subjects' interests in life. The importance and satisfaction were the same across groups for family and friendships relationships. However women with psychosis showed lesser importance and satisfaction for children and intimate relationships. Religious affiliations were equally distributed across groups, with $54 \%$ of Christians, $15 \%$ of other religions (Islam, Buddhism, and Judaism) and $31 \%$ without religious affiliation. There was no association between sexual measures and religious affiliation. Forty percent of women declared that religion and/or spirituality impacted on their sexuality, essentially through guidelines of sexual behaviors, yet without differences across groups.

Women with psychosis gave more importance to religion in their lives, but not to spirituality. For those women, there were no statistically signification associations between sexual measures and the importance of religion and spirituality, but a trend between the importance of religion and individual sexual desire ( $\rho=.47$ individual SDS, $p=.06)$ and sexual dysfunction (IMS $\rho=.46, p=.07$ ). For healthy women, the sexual desire was inversely associated with the importance of religion $(\rho=-.54$ FSFI, $p<.05 ; \rho=-.49$ individual SDS, $p<.03 ; \rho=-.65$ dyad SDS, $p<.01$ ) and with the importance of spirituality $(\rho=-.68$ dyad SDS, $p<.01)$. The sexual dysfunction was associated with the importance of religion (IMS $\rho=.54, p<.05$ ) and with the importance of spirituality (IMS $\rho=.54, p<.05$ ).

\section{Sexuality and Psychosis}

Open questions showed that more than half of the women reported that their psychiatric disorder had an influence on their sexual life (five patients reported a reduction of desire due to medication or illness; two reported a fear of sexuality, two a low sexual-self-esteem due to the illness, and one the lack of adequate housing); reciprocally, $28 \%$ thought that their sexual life impacted on their psychotic 
Table 3 Importance and Satisfaction for Social Relationships

\begin{tabular}{|c|c|c|c|c|c|c|c|c|}
\hline & \multicolumn{2}{|c|}{ Women with Psychosis $(\mathrm{n}=18)$} & \multicolumn{2}{|c|}{ Controls $(\mathrm{n}=21)$} & \multicolumn{2}{|c|}{ Total $(\mathrm{n}=39)$} & \multirow[t]{2}{*}{ Stat. } & \multirow[t]{2}{*}{$p$} \\
\hline & Mean & SD & Mean & SD & Mean & SD & & \\
\hline \multicolumn{9}{|c|}{ Social relationships } \\
\hline \multicolumn{9}{|l|}{ Friendship } \\
\hline Importance $^{\mathrm{a}}$ & 3.0 & 1.3 & 3.3 & .9 & 3.2 & 1.1 & -.55 & .58 \\
\hline Satisfaction $^{\mathrm{b}}$ & 3.0 & .9 & 3.2 & .8 & 3.1 & .9 & -.73 & .47 \\
\hline \multicolumn{9}{|l|}{ Family } \\
\hline Importance & 3.2 & .6 & 3.3 & .8 & 3.2 & .7 & -.84 & .40 \\
\hline Satisfaction & 2.8 & .7 & 2.9 & .9 & 2.9 & .8 & -.36 & .72 \\
\hline \multicolumn{9}{|l|}{ Children } \\
\hline Importance & 2.7 & 1.1 & 3.4 & 1.0 & 3.1 & 1.1 & -2.36 & .02 \\
\hline Satisfaction & 2.9 & 1.0 & 3.5 & .6 & 3.2 & .8 & -2.02 & .04 \\
\hline \multicolumn{9}{|c|}{ Intimate relationships } \\
\hline Importance & 2.6 & 1.0 & 3.3 & .7 & 3.0 & .9 & -2.29 & .02 \\
\hline Satisfaction & 2.1 & .9 & 2.9 & .9 & 2.5 & 1.0 & -2.76 & .01 \\
\hline \multicolumn{9}{|l|}{ Religion } \\
\hline Importance & 2.3 & 1.5 & 1.2 & 1.2 & 1.7 & 1.5 & -2.45 & .01 \\
\hline \multicolumn{9}{|l|}{ Spirituality } \\
\hline Importance & 1.9 & 1.4 & 1.8 & 1.5 & 1.8 & 1.4 & -.39 & .70 \\
\hline
\end{tabular}

${ }^{\text {a }}$ Importance: range from 0 (of no importance) to 4 (essential)

${ }^{\mathrm{b}}$ Satisfaction: range from 0 (of no satisfaction) to 4 (completely satisfied)

disorder for various reasons (two reported an improvement of their mental health through intimate relationships, one attributes her psychotic disorder to sexual abuse during childhood and two reported an increase of paranoid delusion in relation with sexuality).

Only $22 \%$ of patients declared having talked to their psychiatrist about their sexuality, none of those being fully at ease. Only $22 \%$ of patients declared they would be fully embarrassed to broach this topic with their psychiatrist.

\section{Discussion}

We found in this study that, contrary to common knowledge, women with psychosis appear to feature dyadic and individual desire similar to healthy women of comparable age. Yet, women with psychosis had less frequent sexual activity and less satisfaction in sexual function itself, both in the psychological aspects of sexuality and in intimate relationships. Sexual function for this population was related to factors such as past emotional or sexual abuse. Patients' psychiatrists had talked about this issue in a minority of cases.

\section{Desire and Sexual Function}

The fact that desire appears to be unaltered in a population of women with psychosis, most of them being under antipsychotic medications, represents the most relevant finding. Our data contradict those of Fan et al. (2007) who studied patients with psychosis and found an altered libido (e.g. the "biological" side of desire) both for men (79\%) and women $(65 \%)$. Their assessment involved a subscale for desire. Yet their research did not involve a comparison group, but only a confrontation of results with cut-off scores for their questionnaire. Also female patients in their sample were slightly older (42 yo vs. 39 yo in our sample).

Our data on sexual function reveal numerous difficulties for sexual activities themselves, for the psychological issues related to this aspect (e.g. sexual esteem, motivation, anxiety) and for the intimate relationships into which sexual practices is embedded. This is in accordance with Kelly and Conley's review (Kelly and Conley 2004), which underscores that people with schizophrenia engage in less sexual activity and feature multiple aspects of sexual dysfunctions. These authors underscore the need for a better and standardized assessment of sexual function in this population, as studies to date used many different questionnaires. Our results on the MSQ detailing the psychological aspects of sexuality can be compared with Peitl et al. study (2009) on sexual self-perception showing normal readiness for sexual activity yet higher negative emotionality and sexual incompetence in patients with schizophrenia, as compared with healthy volunteers. Mac Donald et al. (2003) study showed in a comparison of schizophrenic patients with healthy controls that women 
had fewer sexual intercourses and a lesser (yet not significant) desire for sex. Factors associated with dysfunction in women were higher negative symptoms and general psychopathological scores. Contrary to our study, positive symptoms were not associated with increase of sexual activity. This discrepancy may be related to the fact that their population could be more stabilized than ours, i.e. preventing the possibility of statistical correlations (an assumption impossible to verify due to the fact that PANSS subscores were not detailed in their study). Also in this study patients completed a quite short survey of ten questions, with only one on this issue ("How frequently would you like to have sexual intercourse"). Hence this method was likely to involve an under-evaluation of a complex concept such as desire.

We also found that only a minority of patients had discussed the issue of sexuality with their clinicians, hence preventing them to get any help for this. This may explain why the sexual desire of women's with psychosis is completely underestimated, as our data suggest. Literature confirms that the intimate sexual and interpersonal needs of women with psychosis remain marginalized and neglected (McCann 2010; Lewis and Scott 1997; Ostman and Bjorkman 2013), while patients would appreciate to discuss this issue (Lewis and Scott 1997).

\section{Sexual Function and Past Abuse}

In our results women with psychosis were more likely to have suffered from abuse. This was associated to some extent with sexual dysfunction. Literature shows an association between various kinds of abuse and risk for schizophrenia (Read et al. 2005) and bad outcome (Sperry and Gilbert 2005). Gearon et al. (2003) found that $61 \%$ of women with psychosis and substance disorder had suffered from sexual abuse. The more severe the abuse, the more likely women with psychosis feature suicidal behaviors, social impairment and positive symptoms (Schenkel et al. 2005).

\section{The Influence of Psychosis on Sexuality}

More than half of women with psychosis studied here report an influence of their disorder on their sexual life. Overall the psychiatric condition of these women is likely to alter their sexuality. This could be related to the wellestablished deficit in social cognition observed in patients suffering from schizophrenia. Indeed, in this population, social cognitions' impairment is characterized notably by the theory of mind's alterations and by deficits in social perception, social knowledge and perception of emotions (Savla et al. 2013), which may influence sexual behavior and desire. Yet many other factors are likely to alter the desire of women with psychosis. Indeed this parameter relies on other psychological factors (physical attraction, sexual fantasies, love feeling) physiological ones (e.g. hormonal status, medications, physical stimulation), and social ones (aspirations, self and social stigma, social norms...) (Kaplan 1995; Levine 2003). Most, if not all, of those factors are likely to be involved in women with psychosis. In their qualitative research on five women with psychosis Ostman and Björkman (2013) described this negative effect of illness well, e.g. through stigma, medication related weight gain and the difficulties of partners to cope with patients' symptoms.

\section{Sexuality and Religion and Spirituality}

For the control group, the relationships elicited between religion and sexuality are in line with the literature. Indeed, the importance of religion is associated for women in general populations with less permissive attitudes toward and lower rates of premarital sexual activity, extramarital affairs, number of sexual partners, high-risk sexual activity (Koenig et al. 2001) and lower sexual desire (Woo et al. 2012). The higher use of spiritual and religious coping by people with severe mental disorders is nowadays a wellknown phenomenon (Mohr et al. 2013), that it also elicited in our sample of women with psychosis. To our knowledge, no study investigates relationships between religion, spirituality and sexuality specifically for women with psychosis. Our results show that, for those women, the trend is the same for the association between importance of religion and higher sexual dysfunction than for healthy control. Yet an unexpected result is the opposite direction of the association between sexual desire and the importance of religion. This area would need further investigations.

\section{Limitations}

This study has several limitations, which prevent to some extent its external validity. First, values and norms related to sexuality and sexual practices are deeply grounded in cultural aspects. Hence these results need to be considered taking into account the occidental setting where it took place, which may influence subjects' representations and practice. The small number of subjects has to be considered, even if this sample allowed us to find many significant differences across groups. Also this sample was characterized by a rather high number of patients declining to enroll, that bringing some possibility of bias. This high number of subjects' refusals to participate is in accordance with other papers on this topic (Ostman and Bjorkman 2013), this phenomenon being probably due to the understandable difficulties women may have to talk about sexuality in a research setting. However, overall, to our 
knowledge, no other paper covers a comparable number of subjects with such an in-depth investigation.

\section{Clinical Implications}

Despite these limitations, we were able to find that even if sexual desire may be preserved in this population of women with psychosis, a lot of problems are present in the concrete aspects of intimate relationships and sexual activities, that being possibly related-among other causes-to past abuse, symptoms and/or (self) stigma. Furthermore, many patients do not have any sexual activities.

Overall these results allow challenging the stigmatizing vision of a widespread impairment in the sexuality of women with severe mental disorder: rather, it appears that the desire and the phantasms related are fully present. Hence the problem for this population may not be as overwhelming as it may appear, being rather due to a lack of the cognitive abilities, the social skills and the interpersonal context allowing an implementation of a meaningful sexuality.

Therefore this issue should be a justifiable concern for mental health professionals, particularly when considering that a vast majority of patients may be willing to address it with their clinicians. This unmet need should foster the implementation of trainings for mental health professionals, which should help patients to develop greater competence in this issue. Alternatively, referral to "marital therapy" and/or sexological treatment should be encouraged when appropriate. These goals should be grounded on the fact that, as suggested by our qualitative findings, severe and long lasting psychiatric disorders may have an influence on sexual life, but conversely sexual problems may impact on the course of psychotic disorders.

Conflict of interest On behalf of all authors, the corresponding author states that there is no conflict of interest.

\section{References}

Affleck, J. W., \& McGuire, R. J. (1984). The measurement of psychiatric rehabilitation status. A review of the needs and a new scale. British Journal of Psychiatry, 145, 517-525.

Basson, R. (2005). Women's sexual dysfunction: Revised and expanded definitions. CMAJ, 172, 1327-1333.

Basson, R. (2006). Sexual desire and arousal disorders in women. New England Journal of Medicine, 354, 1497-1506.

Bernstein, D. P., Stein, J. A., Newcomb, M. D., Walker, E., Pogge, D., Ahluvalia, T., et al. (2003). Development and validation of a brief screening version of the Childhood Trauma Questionnaire. Child Abuse and Neglect, 27, 169-190.

Brohan, E., Clement, S., Rose, D., Sartorius, N., Slade, M., \& Thornicroft, G. (2013). Development and psychometric evaluation of the Discrimination and Stigma Scale (DISC). Psychiatry Research, 208, 33-40.
Bushong, M. E., Nakonezny, P. A., \& Byerly, M. J. (2011). Subjective quality of life and sexual dysfunction in outpatients with schizophrenia or schizoaffective disorder. Journal of Sex and Marital Therapy, 39, 336-346.

Dickson, R. A., \& Dawson, D. T. (1998). Olanzapine and pregnancy. Canadian Journal of Psychiatry, 43, 196-197.

Fan, X., Henderson, D. C., Chiang, E., Briggs, L. B., Freudenreich, O., Evins, A. E., et al. (2007). Sexual functioning, psychopathology and quality of life in patients with schizophrenia. Schizophrenia Research, 9, 119-127.

Friedman, G., \& Harrison, G. (1984). Sexual histories, attitudes, and behavior of schizophrenic and "normal" women. Archives of Sexual Behavior, 13, 555-567.

Gearon, J. S., Kaltman, S. I., Brown, C., \& Bellack, A. S. (2003). Traumatic life events PTSD among women with substance use disorders and schizophrenia. Psychiatric Services, 54, 523-528.

Giles, J. (1958). The nature of sexual desire. Westport, Connecticut: Praeger.

Goldman, H. H., Skodol, A. E., \& Lave, T. R. (1992). Revising axis V for DSM-IV: A review of measures of social functioning. American Journal of Psychiatry, 149, 1148-1156.

Johnson, A. M. (1994). Condoms and HIV transmission. New England Journal of Medicine, 331, 391-392.

Kaplan, H. S. (1995). The sexual desire disorders. New-York: Brunner-Routledge.

Kay, S. R., Opler, L. A., \& Fiszbein, A. (1992). Positive and negative syndrome scale. New-York: Multi-Health Systems Inc.

Kelly, D. L., \& Conley, R. R. (2004). Sexuality and schizophrenia: A review. Schizophrenia Bulletin, 30, 767-779.

Koenig, H. G., McCullough, M. E., \& Larson, D. B. (2001). Handbook of religion and health (pp. 371-380). Oxford: Oxford University Press.

Levine, S. B. (2003). The nature of sexual desire: A clinician's perspective. Archives of Sexual Behavior, 3, 279-285.

Lewis, J., \& Scott, E. (1997). The sexual education needs of those disabled by mental illness. Psychiatric Rehabilitation Journal, $21,164-167$.

MacDonald, S., Halliday, J., MacEwan, T., Sharkey, V., Farrington, S., Wall, S., et al. (2003). Nithsdale Schizophrenia Surveys 24: Sexual dysfunction. Case-control study. The British Journal of Psychiatry, 182, 50-56.

McCann, E. (2010). Investigating mental health service user views regarding sexual and relationship issues. Journal of Psychiatric and Mental Health Nursing, 17, 251-259.

Miller, J. L., \& Finnerty, M. (1996). Sexuality, pregnancy, and childrearing among women with schizophrenia-spectrum disorders. Psychiatric Services (Washington, D. C.), 47, 502-506.

Mohr, S., Borras, L., Nolan, J., Gillièron, C., Brandt, P.-Y., Eytan, A., et al. (2013). Spirituality and religion in outpatients with schizophrenia: A multi-site comparative study of Switzerland, Canada and the United States. International Journal of Psychiatry in Medicine, 44, 29-52.

Neumann, N. U., \& Frasch, K. (2001). Olanzapine and pregnancy: 2 case reports. Nervenarzt, 72, 876-878.

Ostman, M., \& Bjorkman, A. C. (2013). Schizophrenia and relationships: The effect of mental illness on sexuality. Clinical Schizophrenia \& Related Psychoses, 7, 20-24.

Peitl, M. V., Rubesa, G., Peitl, V., Ljubicic, D., \& Pavlovic, E. (2009). Aspects of sexual self-perception in schizophrenic patients. The European Journal of Psychiatry, 23, 37-46.

Raja, M., \& Azzoni, A. (1993). Sexual behavior and sexual problems among patients with severe chronic psychosis. European Psychiatry, 18, 70-76.

Read, J., Van Os, J., Morrison, A. P., \& Ross, C. A. (2005). Childhood trauma, psychosis and schizophrenia: a literature 
review with theoretical and clinical implications. Acta Psychiatrica Scandinavica, 112, 330-350.

Rosen, R., Brown, C., Heiman, J., Leiblum, S., Meston, C., Shabsigh, R., et al. (2000). The Female Sexual Function Index (FSFI): a multidimensional self-report instrument for the assessment of female sexual function. Journal of Sex and Marital Therapy, 26, 191-208.

Savla, G. N., Vella, L., Amstrong, C. C., Penn, L. D., \& Twamley, E. W. (2013). Deficits in domains of social cognition in schizophrenia: A meta-analysis of the empirical evidence. Schizophrenia Bulletin, 39, 979-992.

Schenkel, L. S., Spaulding, W. D., DiLillo, D., \& Silverstein, S. M. (2005). Histories of childhood maltreatment in schizophrenia: relationships with premorbid functioning, symptomatology, and cognitive deficits. Schizophrenia Research, 76, 273-286.

Seagraves, R. T. (1992). Overview of sexual dysfunction complicating the treatment of depression. Journal of Clinical Psychiatry, $10,4-10$

Seeman, M. V. (2013). Clinical interventions for women with schizophrenia: pregnancy. Acta Psychiatrica Scandinavica, 127, 12-22.

Sheehan, D. V., Lecrubier, Y., Sheehan, K. H., Amorim, P., Janavs, J., Weiller, E., et al. (1998). The Mini-International Neuropsychiatric
Interview (MINI): the development and validation of a structured diagnostic psychiatric interview for DSM-IV and ICD-10. Journal of Clinical Psychiatry, 59, 34-57.

Sperry, D. M., \& Gilbert, B. O. (2005). Child peer sexual abuse: Preliminary data on outcomes and disclosure experiences. Child Abuse and Neglect, 29, 889-904.

Thompson, K., Kulkarni, J., \& Sergejew, A. A. (2000). Reliability and validity of a new Medication Adherence Rating Scale (MARS) for the psychoses. Schizophrenia Research, 42, 241-247.

Thornicroft, G., Brohan, E., Rose, D., Sartorius, N., \& Leese, M. (2009). INDIGO Study Group. Global pattern of experienced and anticipated discrimination against people with schizophrenia: A cross-sectional survey. Lancet, 373, 408-415.

Tremblay, S., \& Roussy, L. (2002). Psychometric evaluation of the Sexual Desire Scale (SDS). Science et Comportement, 28, 203-222.

Woo, J. S., Morshedian, N., Brotto, L. A., \& Gorzalka, B. B. (2012). Sex guilt mediates the relationship between religiosity and sexual desire in East Asian and Euro-Canadian college-aged women. Archives of Sexual Behavior, 41, 1485-1495.

World Health Organization (WHO). (1993). Classification statistique internationale des maladies et des problèmes de santé connexes. Genève: Organisation Mondiale de la Santé. 